\title{
ON THE HILBERT SCHEME OF VARIETIES DEFINED BY MAXIMAL MINORS
}

\author{
DANiele FAenzi And Maria Lucia FAnia
}

\begin{abstract}
We compute the dimension of the Hilbert scheme of subvarieties of positive dimension in projective space which are cut by maximal minors of a matrix with polynomial entries.
\end{abstract}

\section{Introduction}

A determinantal subvariety $X$ of the projective space $\mathbb{P}^{n}$ is the locus defined by the vanishing of all minors of a given order of a matrix $M$ of homogeneous polynomials. Many classical varieties can be constructed in this way, for instance Segre and Veronese varieties, rational normal scrolls, Palatini scrolls, Bordiga varieties and so forth. The literature on the subject is rather vast, let us refer to the monographs $[2,15,16,21]$ and $[8$, Lecture 9] for more on these classical objects.

If one attempts to parametrize all determinantal varieties of a given type (i.e., for fixed degrees of the entries of $M$ ), a first step is to look at $[X]$ as a point of a component $\mathscr{H}$ of the Hilbert scheme of subscheme in $\mathbb{P}^{n}$, and study to what extent the family of determinantal varieties fills in $\mathscr{H}$. In this spirit, Ellingsrud proved in [6] that determinantal varieties defined by maximal minors, in case of codimension 2 and dimension $\geq 1$ are unobstructed and their family is open and dense in $\mathscr{H}$. In the series of papers [12-14], the same behavior was established in many more cases, leading to conjecture that this phenomenon should be general.

The goal of this paper is to prove this conjecture in general, for determinantal varieties defined by maximal minors, in the range of dimension at least 1 and codimension at least 2 .

To state our main result properly, we adopt now a more precise language. Given integers $\alpha_{1} \leq \cdots \leq \alpha_{a}, \beta_{1} \leq \cdots \leq \beta_{b}$, and assuming $a \geq b$, we consider a matrix $M=\left(M_{i, j}\right)$ of homogeneous polynomials of degree $\alpha_{j}-\beta_{i}$ in $n+1$ variables, and we let $X$ be defined by all minors of order $b$ of $M$. The subvariety $X$ sits in $\mathbb{P}^{n}$, and we let $p(t)$ be the Hilbert polynomial of $X$. Let us denote by $[X]$ the class of $X$ in the Hilbert

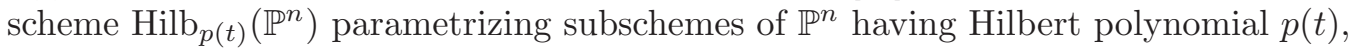
and by $\mathscr{H}$ the irreducible component (or their union if there are more than one) of $\operatorname{Hilb}_{p(t)}\left(\mathbb{P}^{n}\right)$ containing $[X]$. To parametrize all varieties defined by maximal minors, we define the bundles $\mathscr{A}=\oplus_{j=1, \ldots, a} \mathscr{O}_{\mathbb{P}^{n}}\left(-\alpha_{j}\right)$ and $\mathscr{B}=\oplus_{i=1, \ldots, b} \mathscr{O}_{\mathbb{P} n}\left(-\beta_{i}\right)$, and we

Received by the editors July 19, 2013.

2000 Mathematics Subject Classification. Primary 14M12, 14C05; Secondary 14J10, 14 M10.

Key words and phrases. determinantal subvariety, maximal minors, Hilbert scheme. 
consider the vector space $\mathbf{W}$ and the algebraic group $\mathbf{G}$

$$
\mathbf{W}=\operatorname{Hom}_{\mathbb{P}^{n}}(\mathscr{A}, \mathscr{B}), \quad \mathbf{G}=\operatorname{Aut}_{\mathbb{P}^{n}}(\mathscr{A}) \times \operatorname{Aut}_{\mathbb{P}^{n}}(\mathscr{B}) .
$$

A matrix $M$ corresponds this way to an element $\phi$ of $\mathbf{W}$, and the associated variety $X$ is the first degeneracy locus of $\phi$, hence we set $M=M_{\phi}, X=X_{\phi}$. An element $\rho=(g, h) \in \mathbf{G}$ acts on $\mathbf{W}$ by $\rho . \phi=g \circ \phi \circ h^{-1}$. Of course, all elements in the $\mathbf{G}$-orbit $[\phi]$ of $\phi$ give the same degeneracy locus $\phi$, since the ideal generated by minors of a given order is invariant under change of basis. There exists an open subset $\mathbf{W}^{\circ}$ of $\mathbf{W}$ such that $\mathbf{W}^{\circ} / \mathbf{G}$ is a generically smooth irreducible variety that we denote by $\mathscr{Y}$.

We consider thus the natural rational map

$$
F: \mathscr{Y} \rightarrow \mathscr{H}, \quad F:[\phi] \mapsto\left[X_{\phi}\right]
$$

The main result of this note is the following analysis of the rational map $F$.

Theorem. Choose integers $n, b \leq a-1, \alpha_{1} \leq \cdots \leq \alpha_{a}$ and $\beta_{1} \leq \cdots \leq \beta_{b}$, satisfying the following numerical condition:

$$
\alpha_{i} \geq \beta_{i+1}, \forall i=1, \ldots, b-1, \quad \text { and } \alpha_{i}>\beta_{i} \text {, for some } i=1, \ldots, b .
$$

If $\phi$ is general enough, then

$$
\operatorname{dim}\left(X_{\phi}\right)=n+b-a-1, \quad \operatorname{codim}\left(\operatorname{Sing}\left(X_{\phi}\right), X_{\phi}\right) \geq 3 .
$$

Moreover the following holds:

(i) if $n+b-a-1 \geq 1$, then $F$ is generically finite, so $\operatorname{dim}(\operatorname{Im}(F))=\operatorname{dim}(\mathscr{Y})$;

(ii) if $n+b-a-1 \geq 2$, then $F$ is also dominant, in particular $\mathscr{H}$ is an irreducible, generically smooth variety and $\operatorname{dim}(\mathscr{H})=\operatorname{dim}(\mathscr{Y})$; and

(iii) if $\alpha_{1}>\beta_{b}$ and $n+b-a-1 \geq 2$, then $F$ is birational.

As recalled above, this result was motivated by a conjecture of Kleppe and MiróRoig, see [14, Conjecture 4.2], rooted in early work of Ellingsrud. This conjecture is solved by part (ii) above.

What we show is in fact stronger since (iii) proves uniqueness of determinantal representations in the range $\alpha_{1}>\beta_{b}$. Further, part (i) above addresses the positivedimensional range of [14, Conjectures 4.1]. Our result thus completes [11-14], where these conjectures are addressed for several ranges of the $\alpha_{j}$ 's and $\beta_{i}$ 's.

One should be aware that the dimension $\operatorname{dim}(\mathscr{Y})$ can be calculated explicitly as a function of the $\alpha_{j}, \beta_{i}$ 's and $n$. Indeed, we set $c=a-b+1$, and, according to [14], we define $\lambda_{c}$ as

$$
\begin{aligned}
\lambda_{c}= & \sum_{\substack{j=1, \ldots, a \\
i=1, \ldots, b}}\left(\begin{array}{c}
\alpha_{j}-\beta_{i}+n \\
n
\end{array}\right)+\left(\begin{array}{c}
\beta_{i}-\alpha_{j}+n \\
n
\end{array}\right) \\
& -\sum_{i, j=1, \ldots, a}\left(\begin{array}{c}
\alpha_{i}-\alpha_{j}+n \\
n
\end{array}\right)-\sum_{i, j=1, \ldots, b}\left(\begin{array}{c}
\beta_{i}-\beta_{j}+n \\
n
\end{array}\right)+1 .
\end{aligned}
$$


Further we define, for $i=3, \ldots, c$ the integers

$$
\ell_{i}=\sum_{j=1, \ldots, b+i-1} \alpha_{j}-\sum_{i=1, \ldots, b} \beta_{i}, \quad h_{i-3}=2 \alpha_{b+i-1}-\ell_{i}+n .
$$

Finally, for $i=0, \ldots, c-3$, the integers $K_{i+3}$ are defined by

$$
\sum_{\substack{r+s=i \\
r, s \leq 0}} \sum_{\substack{1 \leq i_{1}<\cdots<i_{r} \leq b+i+1, 1 \leq j_{1} \leq \cdots \leq j_{s} \leq b}}(-1)^{i-r}\left(\begin{array}{c}
h_{i}+\alpha_{i_{1}}+\cdots+\alpha_{i_{r}}+\beta_{j_{1}}+\cdots+\beta_{j_{s}} \\
n
\end{array}\right) .
$$

In these terms, the dimension of $\mathscr{Y}$ is

$$
\operatorname{dim}(\mathscr{Y})=\lambda_{c}+K_{3}+\cdots+K_{c} .
$$

Our theorem thus says that the closure of $\mathscr{H}$ in $\operatorname{Hilb}_{p(t)}\left(\mathbb{P}^{n}\right)$ is an irreducible variety of dimension $\lambda_{c}+K_{3}+\cdots+K_{c}$, and in fact an irreducible component of $\operatorname{Hilb}_{p(t)}\left(\mathbb{P}^{n}\right)$ if $\operatorname{dim}\left(X_{\phi}\right)=n+b-a-1 \geq 2$. The following addresses [11, Conjecture 3.2].

Corollary. Let $b \leq a-1, d \geq 1$ be integers and set $\alpha_{j}=d, \beta_{i}=0$ for all $i, j$, and assume $\operatorname{dim}\left(X_{\phi}\right) \geq 2$, i.e., $n+b-a-1 \geq 2$. Then the map $F$ is birational. In particular, $\mathscr{H}$ is an irreducible, generically smooth variety of dimension

$$
\operatorname{dim}(\mathscr{H})=a b\left(\begin{array}{c}
n+d \\
n
\end{array}\right)-a^{2}-b^{2}+1 .
$$

The result above is related to $[7,19]$, where the Hilbert scheme of Palatini scrolls is described. Shortly before submitting our paper, we learned of a preprint by Kleppe [10], addressing very similar questions.

1.1. Structure of the paper. In Section 2, we review some basic material. In Section 3, we calculate the sections of the normal sheaf of $X_{\phi}$, whereby giving an estimate on the dimension of $\mathscr{H}$. Section 4 contains our main lemmas on the fibers of the map $F$, and the proof of the main result.

1.2. Notations and conventions. Let $\boldsymbol{k}$ be an algebraically closed field, $n \geq 2$ be an integer and let $V$ be an $(n+1)$-dimensional vector space over $\boldsymbol{k}$. We consider the projective space $\mathbb{P}^{n}$ of one-dimensional quotients of $V$. Under this convention, we have, for any $d \geq 0$, a natural identification $\mathrm{H}^{0}\left(\mathbb{P}^{n}, \mathscr{O}_{\mathbb{P}^{n}}(d)\right) \cong S^{d} V$, the $d$ th symmetric power of $V$.

If $Z$ is a variety equipped with a morphism $f: Z \rightarrow \mathbb{P}^{n}$, the symbol $H_{Z}$ will denote $f^{*}\left(H_{\mathbb{P} n}\right)$, with $H_{\mathbb{P} n}=c_{1}\left(\mathscr{O}_{\mathbb{P} n}(1)\right)$. Given a morphism $\phi$ of vector bundles on a variety $Z, \mathrm{D}_{k}(\phi)$ will denote the locus consisting of the points $z$ of $Z$ such that $\phi_{z}$ has rank at most $k$, so $\mathrm{D}_{k}(\phi)$ is cut locally by all $(k+1)$-minors of a matrix defining $\phi$.

We will write $R=k\left[x_{0}, \ldots, x_{n}\right] \cong S V$ for the polynomial ring. If $Z$ is a subvariety of $\mathbb{P}^{n}$, we denote by $R_{Z}$ its coordinate ring. We write $\tilde{A}$ for the sheafification of a module $A$ over $R_{Z}$. Given a coherent sheaf $\mathscr{E}$ on $Z$ we denote by $\mathrm{H}_{*}^{0}(\mathscr{E})$ the $R_{Z^{-}}$ module of global sections $\bigoplus_{t \in \mathbb{Z}} \mathrm{H}^{0}(Z, \mathscr{E}(t))$. If $\phi: \mathscr{E} \rightarrow \mathscr{E}$ is a morphism of coherent sheaves over $Z$, then $\mathrm{H}_{*}^{0}(\phi)$ will denote the induced morphism of $R_{Z}$-modules of global sections. We write $A^{*}=\operatorname{Hom}_{R_{Z}}\left(A, R_{Z}\right)$ and $\mathscr{E}^{*}=\mathscr{H}_{\mathrm{om}}\left(\mathscr{E}, \mathscr{O}_{Z}\right)$. We will denote by $\mathscr{N}_{Z^{\prime}, Z}$ the normal sheaf of a subvariety $Z^{\prime}$ in $Z$. The shortcut $\mathrm{h}^{i}(X, \mathscr{E})$ will be used for $\operatorname{dim}_{\boldsymbol{k}} \mathrm{H}^{i}(X, \mathscr{E})$. We also use $\mathbb{P}(\mathscr{E})$ for the projective bundle associated with $\mathscr{E}$, and $S^{d} \mathscr{E}$ for $d$ th symmetric power of $\mathscr{E}$. 


\section{Basic constructions}

The material contained in this section is well known, we refer to the monographs $[2,15,16,21]$. Let us fix some notation and keep it throughout the paper. Given integers $b \leq a-1, \alpha_{1} \leq \cdots \leq \alpha_{a}$ and $\beta_{1} \leq \cdots \leq \beta_{b}$, we define

$$
\mathscr{A}=\bigoplus_{j=1, \ldots, a} \mathscr{O}_{\mathbb{P}^{n}}\left(-\alpha_{j}\right), \quad \mathscr{B}=\bigoplus_{i=1, \ldots, b} \mathscr{O}_{\mathbb{P}^{n}}\left(-\beta_{i}\right), \quad \phi: \mathscr{A} \rightarrow \mathscr{B} .
$$

We write $X_{\phi}$ for the first degeneracy locus of $\phi$, so $X_{\phi}=\mathrm{D}_{b-1}(\phi)$, with the schemetheoretic structure given by the $b \times b$ minors of $\phi$. The expected codimension of $X_{\phi}$ in $\mathbb{P}^{n}$ is $\max (n, a-b+1)$. If $\phi$ is general enough, the actual codimension of $X_{\phi}$ is at least this number if and only if (cf. [13])

$$
\alpha_{i} \geq \beta_{i}, \text { for all } i=1, \ldots, b \quad \text { and } \alpha_{i}>\beta_{i} \text {, for some } i=1, \ldots, b .
$$

2.1. The map $F$. Let $\mathscr{A}$ and $\mathscr{B}$ be as above. The following lemma provides a description of the $\mathbf{G}$-orbit space of $\mathbf{W}$ suitable for our purpose.

Lemma 2.1. There is a generically smooth irreducible variety $\mathscr{Y}$ parametrizing generic $\mathbf{G}$-orbits of $\mathbf{W}$, with

$$
\operatorname{dim}(\mathscr{Y})=\lambda_{c}+K_{3}+\cdots+K_{c} .
$$

Proof. The group G is in general non-reductive. However, we will only be interested in some open piece of the orbit space. According to a result of Rosenlicht, see [18], there is a dense open subset $\mathbf{W}^{\circ}$ such that the quotient $\mathbf{W}^{\circ} / \mathbf{G}$ is geometric. Let us denote

$$
\mathscr{Y}=\mathbf{W}^{\circ} / \mathbf{G} \text {. }
$$

This is a generically smooth variety of dimension

$$
\operatorname{dim}(\mathscr{Y})=\operatorname{dim}(\mathbf{W})-\operatorname{dim}(\mathbf{G})+\operatorname{dim}\left(\mathbf{G}_{\phi}\right),
$$

where $\mathbf{G}_{\phi}$ is the stabilizer of a general element $\phi \in \mathbf{W}^{\circ}$. The dimension of $\mathbf{G}_{\phi}$ is computed in $[12,13]$, and we get $(2.2)$.

2.2. Cokernel of a matrix with polynomial entries. Let $\phi: \mathscr{A} \rightarrow \mathscr{B}$ be as above, and assume (2.1). We assume from now on that $X_{\phi}$ has codimension $c=$ $a-b+1$, so

$$
\operatorname{dim}\left(X_{\phi}\right)=n+b-a-1 .
$$

2.2.1. Cokernel sheaf. We define the sheaf

$$
\mathscr{C}_{\phi}=\operatorname{coker}(\phi) .
$$

Note that $\mathscr{C}_{\phi}$ is supported on $X_{\phi}$. Let $i$ denote the embedding of $X_{\phi}$ in $\mathbb{P}^{n}$. Then $\mathscr{C}_{\phi} \cong i_{*}\left(\mathscr{L}_{\phi}\right)$, for a sheaf $\mathscr{L}_{\phi}$ on $X_{\phi}$ of (generic) rank 1 . The sheaf $\mathscr{L}_{\phi}$ is arithmetically Cohen-Macaulay $(\mathrm{ACM})$ on $X_{\phi}$, i.e., $\mathrm{H}_{*}^{0}\left(X_{\phi}, \mathscr{L}_{\phi}\right)$ is a maximal Cohen-Macaulay module over $R_{X_{\phi}}$. In particular, $\mathscr{L}_{\phi}$ is reflexive, hence invertible if $X_{\phi}$ is integral and locally factorial.

Given the sheaf $\mathscr{L}_{\phi}$, we will consider $c_{1}\left(\mathscr{L}_{\phi}\right)$, as a divisor class in $\mathrm{Cl}(X)$ by looking at the zero locus $D_{\phi}$ of $\mathscr{L}_{\phi}(t)$ (where we choose the smallest $t \in \mathbb{Z}$ such that $\left.\mathrm{H}^{0}\left(X_{\phi}, \mathscr{L}_{\phi}(t)\right) \neq 0\right)$. This locus is in fact a determinantal subvariety $X_{\phi_{0}} \subset \mathbb{P}^{n}$ with 
$\phi_{0}: \mathscr{A} \rightarrow \mathscr{B}_{0}$ and $\mathscr{B}_{0}=\mathscr{B} / \mathscr{O}_{\mathbb{P}^{n}}(-t)$. Note also that the class of $D_{\phi}$ determines $\mathscr{L}_{\phi}$. Indeed, we have an exact sequence

$$
0 \rightarrow \mathscr{L}_{\phi}^{*}(-t) \rightarrow \mathscr{O}_{X_{\phi}} \rightarrow \mathscr{O}_{D_{\phi}} \rightarrow 0
$$

and this gives back $\mathscr{L}_{\phi}$ since $\mathscr{L}_{\phi}^{* *} \cong \mathscr{L}_{\phi}$.

2.2.2. Cokernel module. In terms of $R$-modules, we define

$$
A=\bigoplus_{j=1, \ldots, a} R\left(-\alpha_{j}\right), \quad B=\bigoplus_{i=1, \ldots, b} R\left(-\beta_{i}\right) .
$$

So $\phi$ gives a morphism $\mathrm{H}_{*}^{0}(\phi): A \rightarrow B$, whose sheafification is $\phi$. We define

$$
C_{\phi}=\operatorname{coker}\left(\mathrm{H}_{*}^{0}(\phi)\right) \text {. }
$$

This is a graded $R$-module. Considered as $R_{X}$-module, $C_{\phi}$ is a maximal CohenMacaulay module, and we have $C_{\phi} \cong \mathrm{H}_{*}^{0}\left(\mathscr{C}_{\phi}\right)$ and $\tilde{C}_{\phi} \cong \mathscr{C}_{\phi}$, see for instance [15].

2.3. Determinantal subvarieties as complete intersections. For the constructions in this subsection, we refer for instance to [4,21]. Consider the vector bundle $\mathscr{B}=\bigoplus_{i=1, \ldots, b} \mathscr{O}_{\mathbb{P} n}\left(-\beta_{i}\right)$ over the projective space $\mathbb{P}^{n}$ and define the projective bundle

$$
\mathscr{P}=\mathbb{P}(\mathscr{B}) \stackrel{\pi}{\longrightarrow} \mathbb{P}^{n} .
$$

We have the relatively ample line bundle $\mathscr{O}_{\mathscr{B}}(1)$ and we let $P=c_{1}\left(\mathscr{O}_{\mathscr{B}}(1)\right)$. Set $\mathscr{T}_{\mathscr{B}}$ for the relative tangent bundle. Each $\phi \in \mathbf{W}$ gives a section $s_{\phi} \in \mathrm{H}^{0}\left(\mathscr{P}, \pi^{*}(\mathscr{A})^{*}(P)\right)$ in view of

$$
\mathbf{W}=\operatorname{Hom}_{\mathbb{P}^{n}}(\mathscr{A}, \mathscr{B}) \cong \operatorname{Hom}_{\mathscr{P}}\left(\pi^{*}(\mathscr{A}), \mathscr{O} \mathscr{P}(P)\right) \cong \bigoplus_{i, j} S^{\alpha_{j}-\beta_{i}} V .
$$

Denoting by $\mathbb{V}\left(s_{\phi}\right)$ the vanishing locus of $s_{\phi}$, we define the complete intersection subvariety

$$
Y_{\phi}=\mathbb{V}\left(s_{\phi}\right) \subset \mathscr{P} .
$$

To see the relation between $X_{\phi}$ and $Y_{\phi}$, we consider the scheme $\mathbb{P}\left(\mathscr{L}_{\phi}\right)$, and we let $q$ be the natural map $\mathbb{P}\left(\mathscr{L}_{\phi}\right) \rightarrow \mathbb{P}^{n}$. Since $i_{*}\left(\mathscr{L}_{\phi}\right)$ is a quotient of $\mathscr{B}$, we have a natural closed embedding $p: \mathbb{P}\left(\mathscr{L}_{\phi}\right) \hookrightarrow \mathscr{P}$. Set $P_{Y_{\phi}}=\left.P\right|_{Y_{\phi}}$.

The following lemma is certainly well known, although we have not been able to find the precise statement in the literature. However, we provide a proof for the reader's convenience.

Lemma 2.2. Choose any $\phi \in \mathbf{W}$ with $X_{\phi} \neq \emptyset$. Then $\mathbb{P}\left(\mathscr{L}_{\phi}\right) \cong Y_{\phi}$ as schemes, and we have

$$
\mathscr{L}_{\phi} \cong q_{*}\left(\mathscr{O}_{Y_{\phi}}\left(P_{Y_{\phi}}\right)\right), \quad q\left(Y_{\phi}\right)=X_{\phi}
$$

If the further degeneracy locus $\mathrm{D}_{b-2}(\phi)$ is empty, then $q: \mathbb{P}\left(\mathscr{L}_{\phi}\right) \rightarrow \mathbb{P}^{n}$ is an isomorphism onto $X_{\phi}$, so $Y_{\phi} \cong X_{\phi}$.

Proof. The scheme $\mathbb{P}\left(\mathscr{L}_{\phi}\right)$ consists of the pairs $([\xi],[\gamma])$ where $\xi: V \rightarrow \boldsymbol{k}$ represents a one-dimensional quotient of $V$ and the proportionality class of $\gamma$ lies in $\mathbb{P}\left(\mathscr{L}_{\phi, \xi}\right)$. Since $i_{*}\left(\mathscr{L}_{\phi}\right)$ is defined as coker $(\phi)$, we have that $\gamma$ is a quotient of $\mathscr{L}_{\phi, \xi}$ fitting into

$$
\mathscr{A}_{\xi} \stackrel{\phi_{\xi}}{\longrightarrow} \mathscr{B}_{\xi} \rightarrow \mathscr{L}_{\phi, \xi}
$$


Lifting $\gamma$ to a map $\mathscr{B}_{\xi} \rightarrow \boldsymbol{k}$ (still denoted by $\gamma$ ), we get that $\gamma$ is defined on $\mathscr{L}_{\phi, \xi}$ if and only if $\gamma \circ \phi_{\xi}=0$. Clearly, we have

$$
\gamma \circ \phi_{\xi}=0 \Leftrightarrow \gamma\left(\phi_{\xi}(e)\right)=0, \quad \forall e \in \mathscr{A}_{\xi} .
$$

Summing up we have

$$
\mathbb{P}\left(\mathscr{L}_{\phi}\right)=\left\{([\xi],[\gamma]) \mid \gamma\left(\phi_{\xi}(e)\right)=0, \forall e \in \mathscr{A}_{\xi}\right\} .
$$

On the other hand, $Y_{\phi}$ consists of pairs $([\xi],[\gamma])$ such that $\gamma$ is a quotient of $\mathscr{B}_{\xi}$ and $s_{\phi}$ vanishes at $([\xi],[\gamma])$. By definition of $s_{\phi}$, its evaluation $s_{\phi,([\xi],[\gamma])}$ at a pair $([\xi, \gamma])$ is given as the composition

$$
\mathscr{A}_{\xi} \stackrel{\phi_{\xi}}{\longrightarrow} \mathscr{B}_{\xi} \stackrel{\gamma}{\rightarrow} \mathscr{O}_{\xi}(P) \cong \boldsymbol{k} .
$$

Therefore, we have

$$
Y_{\phi}=\left\{([\xi],[\gamma]) \mid s_{\phi,([\xi],[\gamma])}(e)=\gamma\left(\phi_{\xi}(e)\right)=0, \forall e \in \mathscr{A}_{\xi}\right\}
$$

This agrees with (2.4), so our first statement is proved.

To check (2.3) we note that, since $p$ is induced by the projection $\mathscr{B} \rightarrow i_{*}\left(\mathscr{L}_{\phi}\right)$, there is an isomorphism

$$
p^{*}\left(\mathscr{O}_{\mathscr{P}}(P)\right) \cong \mathscr{O}_{\mathscr{L}_{\phi}}(1)
$$

Clearly, we have

$$
q_{*}\left(\mathscr{O}_{\mathscr{L}_{\phi}}(1)\right) \cong \mathscr{L}_{\phi}
$$

This proves (2.3).

To check the last statement, first note that since $\mathscr{L}_{\phi}$ is supported on $X_{\phi}$ the map $q$ takes value in $X_{\phi}$. When $\mathrm{D}_{b-2}(\phi)=\emptyset$, the sheaf $\mathscr{L}_{\phi}$ is locally free of rank one on $X_{\phi}$ by Pragacz [17]. So $\mathbb{P}\left(\mathscr{L}_{\phi}\right) \cong X_{\phi}$.

Example 2.3. Let $\alpha_{j}=1$ for all $j$ and $\beta_{i}=0$ for all $i$. In this case, $\phi$ corresponds to the choice of a 3 -tensor in $\phi \in \boldsymbol{k}^{a} \otimes \boldsymbol{k}^{b} \otimes \boldsymbol{k}^{n+1}$. The variety $X=X_{\phi}$ is cut in $\mathbb{P}^{n}$ by the $b \times b$ minors of a matrix $M_{\phi}$ of linear forms, of size $a \times b$, while $Y=Y_{\phi}$ is a linear section of codimension $a$ in the Segre product $\mathbb{P}^{b-1} \times \mathbb{P}^{n}$. The map $\pi$ is the projection $\mathbb{P}^{b-1} \times \mathbb{P}^{n}$ onto the second factor $\mathbb{P}^{n}$, and its restriction to $Y$ is $q$.

The variety $Y$ is also obtained as $\mathbb{P}\left(\mathscr{M}_{\phi}\right)$, where $\mathscr{M}_{\phi}=s_{*}\left(q^{*}\left(\mathscr{O}_{\mathbb{P}^{n}}(1)\right)\right)$, and we denote by $s$ the projection $Y \rightarrow \mathbb{P}^{b-1}$. The sheaf $\mathscr{M}_{\phi}$ is presented by a matrix $N_{\phi}$, obtained by exchanging the roles of $\boldsymbol{k}^{b}$ and $\boldsymbol{k}^{n+1}$ in the expression of $\phi \in \boldsymbol{k}^{a} \otimes \boldsymbol{k}^{b} \otimes \boldsymbol{k}^{n+1}$, so $N_{\phi}$ reads

$$
\mathscr{O}_{\mathbb{P}^{b-1}}(-1)^{a} \stackrel{N_{\phi}}{\longrightarrow} \mathscr{O}_{\mathbb{P}^{b-1}}^{n+1} \rightarrow \mathscr{M}_{\phi} \rightarrow 0
$$

For instance, if $a=4, b=3, n=6$, then, for general $\phi, X$ is a 4 -fold in $\mathbb{P}^{6}$ with ten singular points, defined by the order-2 minors of $M=M_{\phi}$. On the other hand, $Y$ is a smooth 4-codimensional linear section of $\mathbb{P}^{2} \times \mathbb{P}^{6}$, which is a $\mathbb{P}^{2}$-bundle over $\mathbb{P}^{2}$, obtained by projectivizing $\mathscr{M}=\mathscr{M}_{\phi}$ which is a rank-3 stable vector bundle on $\mathbb{P}^{2}$ with $c_{1}=4$. As such, $\mathscr{M}$ splits over a general line $\ell$ of $\mathbb{P}^{2}$ as $\mathscr{O}_{\ell}(1)^{2} \oplus \mathscr{O}_{\ell}(2)$. This bundle is 
a rank-3 logarithmic bundle associated with ten lines $\left(\ell_{1}, \ldots, \ell_{10}\right)$ of $\mathbb{P}^{2}$, in the sense of [20]. The lines $\ell_{i}$ are jumping lines of $\mathscr{M}$, in the sense that $\left.\mathscr{M}\right|_{\ell_{i}} \cong \mathscr{O}_{\ell_{i}} \oplus \mathscr{O}_{\ell_{i}}(2)^{2}$. Corresponding to the trivial summand of $\left.\mathscr{M}\right|_{\ell_{i}}$, there is a section the ruled 3 -fold $S_{i}=\mathbb{P}\left(\left.\mathscr{M}\right|_{\ell_{i}}\right)$ which is contracted to a singular point of $X$ in $\mathbb{P}^{6}$. The sheaf $\mathscr{L}_{\phi}$ is locally free of rank 1 away from the ten singular points of $X$ and has rank 2 over these points. The corresponding divisor $D_{i}=q\left(S_{i}\right)$ are Weil divisor, which fail to be Cartier along the ten singular points.

\section{Normal sheaf of a determinantal subvariety}

In order to study the tangent space at $\left[X_{\phi}\right]$ of the Hilbert scheme $\mathscr{H}$, we are now going to compute the normal sheaf of $X_{\phi}$, together with its space of global sections, in the range $(1.1)$, where $X_{\phi}=\mathrm{D}_{b-1}(\phi)$ is given by a general morphism $\phi: \mathscr{A} \rightarrow \mathscr{B}$.

Proposition 3.1. Let $X=X_{\phi}$ and let $\mathscr{N}=\mathscr{N}_{X, \mathbb{P} n}$ be the normal sheaf of $X$ in $\mathbb{P}^{n}$. Assume (1.1), $\operatorname{dim}(X) \geq 2$ and $c=\operatorname{codim}\left(X, \mathbb{P}^{n}\right) \geq 2$. Then we have

$$
\mathrm{h}^{0}(X, \mathscr{N}) \leq \lambda_{c}+K_{3}+\cdots+K_{c}
$$

It will turn out that the above inequality is an equality (cf. proof of the main result, Section 4). The proof of the above proposition will follow from the next lemmas and Proposition 3.5.

Lemma 3.2. In the range (1.1), for $\phi$ general in $\mathbf{W}$, we have

$$
\operatorname{codim}\left(\operatorname{Sing}\left(X_{\phi}\right), X_{\phi}\right) \geq 3
$$

Proof. This follows from [13, Remark 2.7] or equivalently from [3]. Indeed, we construct Chang's filtration of the bundles $\mathscr{B} \supset \mathscr{B}_{1} \supset \cdots \supset \mathscr{B}_{l}$ and $\mathscr{A}_{\supset} \supset \mathscr{A}_{1} \supset \cdots \supset \mathscr{A}_{l}$, as follows. We choose $\mathscr{A}_{1}=\oplus_{j=1}^{r_{1}} \mathscr{O}_{\mathbb{P}^{n}}\left(-\alpha_{j}\right)$ with $r_{1}=\max \left\{j \mid \alpha_{a+1-j} \geq \beta_{b}\right\}$ and $\mathscr{B}_{1}=\oplus_{i=1}^{s_{1}} \mathscr{O}_{\mathbb{P}^{n}}\left(-\beta_{i}\right)$ with $s_{1}=\max \left\{i \mid \beta_{b-i+1}>\alpha_{a-r_{1}}\right\}$. Iterating this procedure, we get the desired filtration, and, since we are assuming $a-b+1 \geq 2$, we obtain by the main theorem of [3] that $\operatorname{codim}\left(X_{\phi}\right)=a-b+1$ and $\operatorname{codim}\left(\operatorname{Sing}\left(X_{\phi}\right), X_{\phi}\right) \geq 3$.

Lemma 3.3. Set $\mathscr{C}=\mathscr{C}_{\phi}$. Then, assuming (2.1), we have

$$
\mathrm{h}^{0}\left(\mathbb{P}^{n}, \mathscr{E} x t_{\mathbb{P}^{n}}^{1}(\mathscr{C}, \mathscr{C})\right) \leq \lambda_{c}+K_{3}+\cdots+K_{c}
$$

Proof. Keep in mind that $X=X_{\phi}$ is an integral ACM subvariety of $\mathbb{P}^{n}$, i.e., $R_{X}$ is a Cohen-Macaulay graded ring. Call $i: X \hookrightarrow \mathbb{P}^{n}$ the embedding. Since $\operatorname{dim}(X) \geq 2$, the fact that $X$ is ACM implies $\mathrm{H}^{1}\left(X, \mathscr{O}_{X}(t)\right)=0$ for all $t \in \mathbb{Z}$, and also $\mathrm{H}^{0}\left(X, \mathscr{O}_{X}\right) \cong \boldsymbol{k}$. Further, recall $\mathscr{C} \cong i_{*}(\mathscr{L})$, where $\mathscr{L}=\mathscr{L}_{\phi}$ is an ACM rank-1 sheaf on $X$. Hence $\mathrm{H}^{1}(X, \mathscr{L}(t))=0$ for all $t \in \mathbb{Z}$. Set $\mathscr{K}=\operatorname{ker}(\phi)$. We have an exact sequence

$$
0 \rightarrow \mathscr{K} \rightarrow \mathscr{A} \rightarrow \mathscr{B} \rightarrow \mathscr{C} \rightarrow 0
$$


By [13, Lemma 3.2], we have

$$
\mathscr{H} 0 m_{\mathbb{P}^{n}}(\mathscr{C}, \mathscr{C}) \cong i_{*}\left(\mathscr{O}_{X}\right) .
$$

Therefore, applying $\mathscr{H}_{o m_{\mathbb{P}^{n}}}(-, \mathscr{C})$ to $(3.1)$, we obtain the long exact sequence

$$
0 \rightarrow i_{*}\left(\mathscr{O}_{X}\right) \rightarrow \mathscr{B}^{*} \otimes \mathscr{C} \stackrel{\psi}{\rightarrow} \mathscr{A}^{*} \otimes \mathscr{C} \rightarrow \mathscr{F} \rightarrow 0
$$

where the sheaf $\mathscr{F}$, defined by the sequence above, is supported on $X$. We have

$$
\mathscr{E} x t_{\mathbb{P}^{n}}^{1}(\mathscr{C}, \mathscr{C}) \subset \mathscr{F}
$$

Then we have

$$
\mathrm{h}^{0}\left(\mathbb{P}^{n}, \mathscr{E} x t_{\mathbb{P}^{n}}^{1}(\mathscr{C}, \mathscr{C})\right) \leq \mathrm{h}^{0}\left(\mathbb{P}^{n}, \mathscr{F}\right)
$$

and we want to show

$$
\mathrm{h}^{0}\left(\mathbb{P}^{n}, \mathscr{F}\right)=\lambda_{c}+K_{3}+\cdots+K_{c} .
$$

Let us assume for the moment that the following claim holds:

Claim 3.4. Whenever $\operatorname{dim}(X) \geq 2$, we have $\mathrm{H}^{1}(X, \operatorname{Im}(\psi))=0$.

Set $f(t)=\mathrm{h}^{0}(X, \mathscr{L}(t))=\mathrm{h}^{0}\left(\mathbb{P}^{n}, \mathscr{C}(t)\right)$. Assuming the above claim, we can write

$$
\mathrm{h}^{0}\left(\mathbb{P}^{n}, \mathscr{F}\right)=\sum_{j=1, \ldots, a} f\left(\alpha_{j}\right)-\sum_{i=1, \ldots, b} f\left(\beta_{i}\right)+1 .
$$

The Buchsbaum-Rim complex associated with $\mathrm{H}_{*}^{0}(\phi)$ gives the graded free resolution of $C_{\phi}$

$$
\begin{aligned}
0 & \rightarrow \wedge^{a} A \otimes S^{c-2} B^{*}(\beta) \rightarrow \cdots \rightarrow \wedge^{b+s+1} A \otimes S^{s} B^{*}(\beta) \rightarrow \\
\cdots & \rightarrow \wedge^{b+1} A(\beta) \rightarrow A \rightarrow B \rightarrow C_{\phi} \rightarrow 0,
\end{aligned}
$$

where we set $\beta=\sum_{i=1, \ldots, b} \beta_{i}$. Put $\mathscr{E}_{s}=\wedge^{b+s+1} \mathscr{A} \otimes S^{s}\left(\mathscr{B}^{*}\right)(\beta)$. Sheafifying (3.3) and computing global sections, we get

$$
\begin{aligned}
f(t)= & \sum_{i=1, \ldots, b}\left(\begin{array}{c}
n-\beta_{i}+t \\
n
\end{array}\right)-\sum_{r=1, \ldots, a}\left(\begin{array}{c}
n-\alpha_{r}+t \\
n
\end{array}\right) \\
& +\sum_{s=0}^{c-2}(-1)^{s} \mathrm{~h}^{0}\left(\mathbb{P}^{n}, \mathscr{E}_{s}(t)\right) .
\end{aligned}
$$

Further, it is easy to compute $\mathrm{h}^{0}\left(\mathbb{P}^{n}, \mathscr{E}_{s}(t)\right)$ as

$$
\sum_{\substack{1 \leq i_{1}<\cdots<i_{a-b-s-1} \leq a \\
1 \leq j_{1} \leq \cdots \leq j_{s} \leq b}}\left(\begin{array}{c}
n-\ell+\alpha_{i_{1}}+\cdots+\alpha_{i_{a-b-s-1}}+\beta_{j_{1}}+\cdots+\beta_{j_{s}}+t \\
n
\end{array}\right) .
$$


Note that, in view of (2.1), the upper term appearing in the binomial coefficient above is strictly bounded above by $-\alpha_{b+1}+t+n$, hence the binomial coefficient vanishes for $t=\beta_{i}$, and $t=\alpha_{i-1}$ with $i \leq b+1$. Then, combining (3.5) and (3.4), we get an expression for $f(t)$, hence for $\mathrm{h}^{0}\left(\mathbb{P}^{n}, \mathscr{F}\right)$ in view of $(3.2)$. Recalling the definition of $\lambda_{c}$ from (1.2) and of the $K_{i}$ 's from (1.3), one now easily gets the desired expression for $\mathrm{h}^{0}\left(\mathbb{P}^{n}, \mathscr{F}\right)$.

Proof of Claim 3.4. If $\operatorname{dim}(X) \geq 3$ the vanishing is clear since $\mathrm{H}^{1}(X, \mathscr{L}(t))=0$ for all $t \in \mathbb{Z}$ and $\mathrm{H}^{2}\left(X, \mathscr{O}_{X}\right)=0$. So we only have to prove the vanishing for $\operatorname{dim}(X)=$ $n+b-a-1=2$. Set $Y=Y_{\phi}$ and recall that $X=q(Y)$. We get the sequence defining $\operatorname{Im}(\psi)$ applying $q_{*}$ to

$$
\left.0 \rightarrow \mathscr{O}_{Y} \rightarrow \pi^{*} \mathscr{B}^{*} \otimes \mathscr{O}_{Y}\left(P_{Y}\right) \rightarrow\left(\mathscr{T}_{\mathscr{B}}\right)\right|_{Y} \rightarrow 0
$$

where $\mathscr{T}_{\mathscr{B}}$ is the relative tangent bundle of $\pi$. So we want

$$
\mathrm{H}^{1}\left(Y,\left.\left(\mathscr{T}_{\mathscr{B}}\right)\right|_{Y}\right)=0 .
$$

To obtain this vanishing, we look at the Koszul complex of $s_{\phi}$ and we tensor it by $\mathscr{T}_{\mathscr{B}}$. The $k$ th term of this complex is $\pi^{*}\left(\wedge^{k} \mathscr{A}\right) \otimes \mathscr{T}_{\mathscr{B}}(-k P)$. Then it suffices to show

$$
\mathrm{H}^{k}\left(\mathscr{P}, \mathscr{T}_{\mathscr{B}}\left((1-k) P+t H_{\mathscr{P}}\right)\right)=0, \quad \text { for } k=1, \ldots, a+1 \text { and } \forall t \in \mathbb{Z} \text {. }
$$

The sequence (3.6) is the restriction of the relative Euler sequence twisted by $\mathscr{O}_{\mathscr{P}}(-P)$

$$
0 \rightarrow \mathscr{O}_{\mathscr{P}}(-P) \rightarrow \pi^{*}\left(\mathscr{B}^{*}\right) \rightarrow \mathscr{T}_{\mathscr{B}}(-P) \rightarrow 0
$$

Recall that we set $\beta=\sum_{i=1, \ldots, b} \beta_{i}$. We will use the natural isomorphisms (see for instance [9, Exercise 8.4, p. 253])

$$
\mathbf{R}^{j} \pi_{*}\left(\mathscr{O}_{\mathscr{P}}(1-\ell) P\right) \cong \begin{cases}S^{1-\ell} \mathscr{B}, & \text { for } j=0 \text { and } \ell \leq 1 \\ \left(S^{\ell-1-b} \mathscr{B}\right)^{*}(\beta), & \text { for } j=b-1, \ell \geq b+1 \\ 0, & \text { for } j \neq 0, b-1\end{cases}
$$

Applying direct image functors $\mathbf{R}^{j} \pi_{*}$ to (3.8) we easily obtain the vanishing of $\mathbf{R}^{j} \pi_{*}\left(\mathscr{T}_{\mathscr{B}}((1-\ell) P)\right)$ for $1 \leq j \leq b-3$ (and obviously for $j \geq b$ ).

Now we need to compute this sheaf for $j=b-1$ and $j=b-2$. For $j=b-2$, we show that it vanishes except for $\ell=b+1$ and for $j=b-1$, we show that it is a direct sum of line bundles and non-zero only for $\ell \geq b-3$. In fact, $\mathbf{R}^{j} \pi_{*}\left(\mathscr{T}_{\mathscr{B}}((1-\ell) P)\right)$ are the kernel and cokernel, respectively, for $j=b-2$ and $j=b-1$, of the induced morphism

$$
\mathbf{R}^{b-1} \pi_{*}\left(\mathscr{O}_{\mathscr{P}}((1-\ell) P) \rightarrow \mathscr{B}^{*} \otimes \mathbf{R}^{b-1} \pi_{*}\left(\mathscr{O}_{\mathscr{P}}((2-\ell) P)\right)\right.
$$


We deduce $\mathbf{R}^{b-2} \pi_{*}\left(\mathscr{T}_{\mathscr{B}}((1-\ell) P)=0\right.$ for $\ell \leq b$. For higher $\ell$, using (3.9), the morphism (3.10) takes the form

$$
\left(S^{\ell-1-b} \mathscr{B}\right)^{*}(\beta) \rightarrow \mathscr{B}^{*} \otimes\left(S^{\ell-2-b} \mathscr{B}\right)^{*}(\beta)
$$

For $\ell=b+2$, this map is an isomorphism, and for all $\ell \geq b+3$ it is injective, since it is just the twisted dual of the obvious multiplication map

$$
\mathscr{B} \otimes S^{\ell-2-b} \mathscr{B} \rightarrow S^{\ell-1-b} \mathscr{B}
$$

Therefore, from injectivity of (3.11) we deduce $\mathbf{R}^{b-2} \pi_{*}\left(\mathscr{T}_{\mathscr{B}}((1-\ell) P)=0\right.$ for $\ell \geq b+2$. We have proved that $\mathbf{R}^{b-2} \pi_{*}\left(\mathscr{T}_{\mathscr{B}}((1-\ell) P)\right)=0$ except for $\ell=b+1$. Moreover in this case, we get $\mathbf{R}^{b-2} \pi_{*}\left(\mathscr{T}_{\mathscr{B}}(-b P)\right) \cong \mathscr{O}_{\mathbb{P}^{n}}(\beta)$. Also, $\mathbf{R}^{b-1} \pi_{*}\left(\mathscr{T}_{\mathscr{B}}((1-\ell) P)\right)=0$ for $\ell \leq b+2$ while for $\ell \geq b+3$ we get that $\mathbf{R}^{b-1} \pi_{*}\left(\mathscr{T}_{\mathscr{B}}((1-\ell) P)\right)$ is the bundle $\left(S^{\ell-2-b, 1} \mathscr{B}\right)^{*}(\beta)$ obtained by plethysm (cf. [21]), which is also a direct sum of line bundles.

We can now conclude by the Leray spectral sequence

$$
\mathrm{H}^{i}\left(\mathbb{P}^{n}, \mathbf{R}^{j} \pi_{*}\left(\mathscr{T}_{\mathscr{B}}((1-i-j) P)\right)\right) \Rightarrow \mathrm{H}^{i+j}\left(\mathscr{P}, \mathscr{T}_{\mathscr{B}}((1-i-j) P)\right) .
$$

Indeed, by the previous analysis, the only terms contributing to $\mathrm{H}^{i+j}\left(\mathscr{P}, \mathscr{T}_{\mathscr{B}}((1-i-\right.$ $\left.\left.j) P+t H_{\mathscr{P}}\right)\right)$ appear for $j=b-2$ or $j=b-1$. Recall also that we only have to treat the case $a=n+b-3$ and $a-b+1 \geq 2$, i.e., $n \geq 4$.

Looking at the case $j=b-1$, we have said that $\mathbf{R}^{j} \pi_{*}\left(\mathscr{T}_{\mathscr{B}}(1-i-j) P\right)$ ) is a direct sum of line bundles for $i+j \geq b+3$, or zero for $i+j \leq b+2$. So we may assume $i \geq b+3-j=4$. Also, $i+j \leq a+1$ implies $i \leq a-b+2=n-1$, so $\mathrm{H}^{i}\left(\mathbb{P}^{n}, \mathbf{R}^{j} \pi_{*}\left(\mathscr{T}_{\mathscr{B}}(1-i-j) P\right)\right)=0$ in this range. Then, (3.7) follows from (3.12).

In case $j=b-2$, in order for $\mathbf{R}^{j} \pi_{*}\left(\mathscr{T}_{\mathscr{B}}(1-i-j) P\right)$ ) to be non-zero (and hence isomorphic to $\left.\mathscr{O}_{\mathbb{P} n}(\beta)\right)$ we must have $i+j=b+1$, which implies $i=3$. Hence $n \geq 4$ gives $i \leq n-1$ and therefore $\mathrm{H}^{i}\left(\mathbb{P}^{n}, \mathscr{O}_{\mathbb{P}^{n}}(\beta)\right)=0$. So (3.7) holds again by (3.12).

Proposition 3.5. Assuming (1.1), we have

$$
i_{*}(\mathscr{N}) \cong \mathscr{E} x t_{\mathbb{P} n}^{1}(\mathscr{C}, \mathscr{C}) .
$$

Proof. We recall the identification $\mathscr{C} \cong i_{*}(\mathscr{L})$, and the natural isomorphism

$$
i_{*}(\mathscr{N}) \cong \mathscr{E} x t_{\mathbb{P} n}^{1}\left(i_{*}\left(\mathscr{O}_{X}\right), i_{*}\left(\mathscr{O}_{X}\right)\right)
$$

We have to provide an isomorphism of the right-hand side with $\mathscr{E} x t_{\mathbb{P}^{n}}^{1}(\mathscr{C}, \mathscr{C})$. In order to obtain it, we consider the cohomological spectral sequence

$$
E_{2}^{p, q}=\mathscr{E} x t_{\mathbb{P} n}^{p}\left(i_{*}\left(\mathscr{O}_{X}\right), i_{*}\left(\mathscr{E} x t_{X}^{q}(\mathscr{L}, \mathscr{L})\right)\right) \Rightarrow \mathscr{E} x t_{\mathbb{P}^{n}}^{p+q}(\mathscr{C}, \mathscr{C})
$$

Let us postpone to the end of the proof the explanation for this formula, and assume it for now. 
Since we have seen that $\mathscr{H} o m_{X}(\mathscr{L}, \mathscr{L}) \cong \mathscr{O}_{X},(3.13)$ this gives the required isomorphism once we prove $\mathscr{E} x t_{X}^{1}(\mathscr{L}, \mathscr{L})=0$. In order to show this vanishing, we recall that $\mathscr{L} \cong q_{*}\left(\mathscr{O}_{Y}\left(P_{Y}\right)\right)$, where we have set $Y=Y_{\phi}$. Projection formula provides a natural isomorphism

$$
\mathscr{E} x t_{X}^{1}(\mathscr{L}, \mathscr{L}) \cong \mathscr{E} x t_{Y}^{1}\left(q^{*}(\mathscr{L}), \mathscr{O}_{Y}\left(P_{Y}\right)\right) .
$$

To show that the right-hand side vanishes, we write the relative dual Euler sequence

$$
0 \rightarrow \Omega_{\phi}\left(P_{Y_{\phi}}\right) \rightarrow q^{*}\left(\mathscr{L}_{\phi}\right) \rightarrow \mathscr{O}_{Y_{\phi}}\left(P_{Y_{\phi}}\right) \rightarrow 0
$$

where the relative cotangent sheaf $\Omega_{\phi}$ is defined as the kernel of the canonical surjection above, and is supported on the locus in $Y_{\phi}$ blown down by $q$. We apply $\mathscr{H}_{0} m_{Y}\left(-, \mathscr{O}_{Y}\left(P_{Y}\right)\right)$ to the exact sequence (3.14). Clearly, $\mathscr{E} x t_{Y}^{1}\left(\mathscr{O}_{Y}\left(P_{Y}\right), \mathscr{O}_{Y}\left(P_{Y}\right)\right)=0$ for $\mathscr{O}_{Y}\left(P_{Y}\right)$ is locally free. Further, $q$ is a birational surjective morphism, and the support $Z_{\phi}$ of $\Omega_{\phi}$ lies over $\mathrm{D}_{b-2}(\phi) \subset$ $\operatorname{Sing}\left(X_{\phi}\right)$, where the fibers of $q$ are generically contained in a $\mathbb{P}^{1}$. Then we have $\operatorname{codim}\left(Z_{\phi}, Y_{\phi}\right) \geq \operatorname{codim}\left(\operatorname{Sing}\left(X_{\phi}\right), X_{\phi}\right)-1 \geq 2$ by Lemma 3.2 . Then we get $\mathscr{E} x t_{Y}^{1}\left(\Omega_{\phi}\left(P_{Y_{\phi}}\right), \mathscr{O}_{Y}\left(P_{Y}\right)\right)=0$, and so $\mathscr{E} x t_{Y}^{1}\left(q^{*}(\mathscr{L}), \mathscr{O}_{Y}\left(P_{Y}\right)\right)=0$ and we are done.

Finally, let us prove (3.13). Let $\mathscr{E}$ be a coherent sheaf on $X$. We consider the functors $\Psi=\mathscr{H} \operatorname{om}_{\mathbb{P} n}\left(i_{*}\left(\mathscr{O}_{X}\right), i_{*}(-)\right): \operatorname{Coh}(X) \rightarrow \operatorname{Coh}\left(\mathbb{P}^{n}\right)$ and $\Phi=\mathscr{H}_{0} m_{X}(\mathscr{E},-):$ $\operatorname{Coh}(X) \rightarrow \operatorname{Coh}(X)$. The composition $\Psi \circ \Phi$, applied to a coherent sheaf $\mathscr{G}$ on $X$, is

$$
\mathscr{H} \operatorname{om}_{\mathbb{P}^{n}}\left(i_{*}\left(\mathscr{O}_{X}\right), i_{*}\left(\mathscr{H} o m_{X}(\mathscr{E}, \mathscr{G})\right)\right) \cong \mathscr{H} o m_{\mathbb{P}^{n}}\left(i_{*}(\mathscr{E}), i_{*}(\mathscr{G})\right) .
$$

To see this isomorphism, we can work locally and replace the map $i$ with the closed embedding $\operatorname{Spec}(A) \rightarrow \operatorname{Spec}(B)$ induced by a surjective map of $\boldsymbol{k}$-algebras $B \rightarrow A$, so that $\mathscr{E}, \mathscr{G}$ should be replaced with finitely generated modules $M, N$ over $A$. Then, to prove $(3.15)$, we have to check

$$
\left.\operatorname{Hom}_{B}(M, N) \cong \operatorname{Hom}_{B}\left(A, \operatorname{Hom}_{A}(M, N)\right)\right) .
$$

To do this, it suffices to send a $B$-morphism $u: M \rightarrow N$ to the map $1_{A} \rightarrow u$, and one easily sees that this gives the desired isomorphism of $B$-modules.

In this setting, Grothendieck's spectral sequence associated with the composition of the two left-exact functors $\Psi$ and $\Phi$, applied after replacing $\mathscr{E}=\mathscr{G}=\mathscr{L}$ and recalling that $\mathscr{C}=i_{*}(\mathscr{L})$, gives the required formula (3.13) in view of (3.15).

The previous lemmas, together with Proposition 3.5, suffice to prove Proposition (3.1).

Example 3.6. Going back to our example of a matrix of size $3 \times 4$ over $\mathbb{P}^{6}$, we see that $\Omega_{\phi}\left(P_{Y_{\phi}}\right)$ is the direct sum of the $\mathscr{O}_{\ell_{i}}(-1)$ for $i=1, \ldots, 10$, and as such is supported in codimension 3 in $Y$.

\section{Fibers of the map $F$}

We will prove here our main result. Again we assume (1.1) and we let $\phi$ be a morphism $\phi: \mathscr{A} \rightarrow \mathscr{B}$ such that $X_{\phi}$ has codimension $c=a-b+1$ hence $\operatorname{dim}\left(X_{\phi}\right)=n-c=$ $n-a+b-1$. 
4.1. Transitiveness on the fibers for fixed cokernel sheaves. The following lemma shows that, once we fix the isomorphism class of the cokernel sheaf $\mathscr{C}_{\phi}$, the group $\mathbf{G}$ operates transitively on the fibers of $F$.

Lemma 4.1. Assume $\operatorname{dim}\left(X_{\phi}\right) \geq 1$ and let $\phi^{\prime}$ be a morphism $\mathscr{A} \rightarrow \mathscr{B}$ such that the sheaves $\mathscr{C}_{\phi}$ and $\mathscr{C}_{\phi^{\prime}}$ are isomorphic. Then there are $g \in \operatorname{Aut}_{\mathbb{P} n}(\mathscr{A})$ and $h \in \operatorname{Aut}_{\mathbb{P} n}(\mathscr{B})$, such that $h \circ \phi=\phi^{\prime} \circ g$.

Proof. We have $\mathscr{C}_{\phi} \cong \mathscr{C}_{\phi^{\prime}}$ and $C_{\phi^{\prime}} \cong C_{\phi}$. The Buchsbaum-Rim complexes associated with $\mathrm{H}_{*}^{0}(\phi)$ and $\mathrm{H}_{*}^{0}\left(\phi^{\prime}\right)$ give graded free resolutions of $C_{\phi^{\prime}} \cong C_{\phi}$. If the resolution associated with $\phi$ is minimal, then so is the one associated with $\phi^{\prime}$, since these resolutions share the same Betti numbers. By the uniqueness of the minimal graded free resolution (see e.g. [5]), we get the desired maps $g \in \operatorname{Aut}_{\mathbb{P}^{n}}(\mathscr{A}) \cong \operatorname{Aut}_{R}(A)$, $h \in \operatorname{Aut}_{\mathbb{P}^{n}}(\mathscr{B}) \cong \operatorname{Aut}_{R}(B)$ with $h \circ \phi=\phi^{\prime} \circ g$.

If the resolution associated with $\phi$ is not minimal, then we have $A \cong A_{1} \oplus A_{2}$ and $B \cong B_{1} \oplus B_{2}$, hence a block decomposition of $\phi$

$$
\phi=\phi_{1} \oplus \phi_{2}=\left(\begin{array}{cc}
\phi_{1} & 0 \\
0 & \phi_{2}
\end{array}\right),
$$

where $\phi_{1}: A_{1} \rightarrow B_{1}$ is minimal and $\phi_{2}: A_{2} \rightarrow B_{2}$ is an isomorphism. Again by the uniqueness of the minimal graded free resolution, we get a decomposition $\phi^{\prime}=\phi_{1}^{\prime} \oplus \phi_{2}^{\prime}$ and isomorphisms $g_{1} \in \operatorname{Aut}_{R}\left(A_{1}\right), h_{1} \in \operatorname{Aut}_{R}\left(B_{1}\right)$ with $h_{1} \circ \phi_{1}=\phi_{1}^{\prime} \circ g_{1}$. Then we can take $g_{2}=\mathrm{id}_{A_{2}}$, and $h_{2}=\phi_{2}^{\prime} \circ \phi_{2}^{-1}$, so setting $g=g_{1} \oplus g_{2}$ and $h=h_{1} \oplus h_{2}$ gives $h \circ \phi=\phi^{\prime} \circ g$.

4.2. Finiteness and uniqueness of determinantal representations. We start with two lemmas that account for the finiteness and the uniqueness of the fiber of the map $F$, i.e., of determinantal representations of a given subvariety $X=X_{\phi}$ of $\mathbb{P}^{n}$. This will lead to the proof of our main result.

Lemma 4.2. If $\operatorname{dim}\left(X_{\phi}\right) \geq 1$, then, up to $\mathbf{G}$-action, there are finitely many $\phi^{\prime} \in \mathbf{W}$ such that

$$
\mathscr{C}_{\phi} \neq \mathscr{C}_{\phi^{\prime}}, \quad X_{\phi}=X_{\phi^{\prime}}
$$

Proof. Let $\phi^{\prime}$ be a morphism $\mathscr{A} \rightarrow \mathscr{B}$ such that $X_{\phi}=X_{\phi^{\prime}}$ and set $X=X_{\phi}$. Then $\phi^{\prime}$ defines a cokernel sheaf $\mathscr{C}_{\phi^{\prime}} \cong i_{*}\left(\mathscr{L}_{\phi^{\prime}}\right)$. Also, $X$ is the image via a map $q^{\prime}$ of $Y_{\phi^{\prime}}=\mathbb{V}\left(s_{\phi^{\prime}}\right)$, according to Lemma 2.2 , and we have $\mathscr{L}_{\phi^{\prime}} \cong q_{*}\left(\mathscr{O}_{Y_{\phi^{\prime}}}\left(P_{Y_{\phi^{\prime}}}^{\prime}\right)\right)$.

Now comes an important point to obtain our result, namely the canonical class of $X_{\phi}$. We set $c_{1}\left(\mathscr{L}_{\phi}\right)=P_{X}, c_{1}\left(\mathscr{L}_{\phi^{\prime}}\right)=P_{X}^{\prime}$, as an element of $\mathrm{Cl}(X)$, and we define the divisor class $H_{\mathscr{P}}=\pi^{*}\left(H_{\mathbb{P}^{n}}\right)$ and its restriction $H_{Y_{\phi}}$ to $Y_{\phi}$. We have (see e.g. [2])

$$
K_{Y_{\phi}} \cong(\ell-n-1) H_{Y_{\phi}}+(a-b) P_{Y_{\phi}}, \quad c_{1}\left(\omega_{X_{\phi}}\right) \cong(\ell-n-1) H_{X_{\phi}}+(a-b) P_{X_{\phi}} .
$$

Therefore

$$
(\ell-n-1) H_{X}+(a-b) P_{X} \equiv(\ell-n-1) H_{X}+(a-b) P_{X}^{\prime}, \quad \text { in } \mathrm{Cl}(X) .
$$


Pulling back to $Y_{\phi}$, we get the equality

$$
(a-b)\left(P_{Y_{\phi}}-q^{*}\left(P_{X}^{\prime}\right)\right) \equiv 0, \quad \text { in } \mathrm{Cl}\left(Y_{\phi}\right) .
$$

We observe that $q^{*}$ gives an isomorphism between $\mathrm{Cl}\left(Y_{\phi}\right)$ and $\mathrm{Cl}(X)$, for $q$ is biregular outside a closed subset of codimension at least 2. Moreover, $P_{Y_{\phi}}$ is a Cartier divisor of $Y_{\phi}$ so the above equality takes place in $\operatorname{Pic}\left(Y_{\phi}\right)$. Note that $a-b \neq 0$ by hypothesis, and that $\operatorname{Pic}\left(Y_{\phi}\right)$ has only finitely many points of order $a-b$. Indeed, this is clear if $\operatorname{dim}\left(Y_{\phi}\right)=1$ for $\operatorname{Pic}\left(Y_{\phi}\right)$ is then smooth, and also if $\operatorname{dim}\left(Y_{\phi}\right) \geq 2$ since in this case $\mathrm{H}^{1}\left(Y_{\phi}, \mathscr{O}_{Y_{\phi}}\right)=\mathrm{H}^{1}\left(X, \mathscr{O}_{X}\right)=0$, so that $\operatorname{Pic}\left(Y_{\phi}\right)$ is reduced to the Nron-Severi group, which is finitely generated.

Then, recalling that $c_{1}\left(\mathscr{L}_{\phi^{\prime}}\right)$ determines $\mathscr{L}_{\phi^{\prime}}$ (see Section 2.2 ), we get that there are only finitely many ways to choose the isomorphism class of $\mathscr{L}_{\phi^{\prime}}$ in such a way that the above equation is satisfied. In other words, there are $\phi_{1}, \ldots, \phi_{r}$ such that $\mathscr{C}_{\phi_{i}} ¥ \mathscr{C}_{\phi_{j}}$ if $i \neq j$, and such that for any other $\phi_{0} \in \mathbf{W}$ we have $\mathscr{C}_{\phi_{0}} \cong \mathscr{C}_{\phi_{i}}$ for one $i=1, \ldots, r$. By Lemma (4.1), this $\phi_{0}$ is taken to $\phi_{i}$ by the action of $\mathbf{G}$, which proves our claim.

Lemma 4.3. Let $\phi$ be a general element of $\operatorname{Hom}_{\mathbb{P}^{n}}(\mathscr{A}, \mathscr{B})$, and assume

$$
\alpha_{1}>\beta_{b}, \quad \operatorname{dim}\left(X_{\phi}\right) \geq 2, \quad c=a-b+1 \geq 2 .
$$

Then, given another morphism $\phi^{\prime}$ with $X_{\phi}=X_{\phi^{\prime}}$, we have $\mathscr{C}_{\phi} \cong \mathscr{C}_{\phi^{\prime}}$.

Proof. Let $X=X_{\phi}$, and consider the divisor $P_{X}$ associated with $\mathscr{C}_{\phi}$. In view of the considerations of the previous lemma, any $\phi^{\prime}$ such that $X_{\phi^{\prime}}=X$ gives a divisor class $P_{X}^{\prime}$ such that

$$
(a-b)\left(P_{Y_{\phi}}-q^{*}\left(P_{X}^{\prime}\right)\right) \equiv 0, \quad \text { in } \operatorname{Pic}\left(Y_{\phi}\right) .
$$

Again $a-b \neq 0$, so that $P_{Y_{\phi}}-q^{*}\left(P_{X}^{\prime}\right)$ is of order $a-b \operatorname{in} \operatorname{Pic}\left(Y_{\phi}\right)$. Now $Y_{\phi}$ is the complete intersection in $\mathscr{P}$, of $a$ (Cartier) divisors, whose classes are

$$
\left(\alpha_{1} H_{\mathscr{P}}+P_{\mathscr{P}}, \ldots, \alpha_{a} H_{\mathscr{P}}+P_{\mathscr{P}}\right) .
$$

Under the hypothesis $\alpha_{1}>\beta_{b}$, all the divisors $\alpha_{j} H_{\mathscr{P}}+P_{\mathscr{P}}$ are very ample on $\mathscr{P}$, indeed the direct image $\pi_{*}\left(\mathscr{O}_{\mathscr{P}}\left(\alpha_{j} H_{\mathscr{P}}+P_{\mathscr{P}}\right)\right)$ decomposes as a direct sum of positive line bundles. Therefore we can argue, by Grothendieck-Lefschetz theorem, that $Y_{\phi}$ is smooth and $\operatorname{Pic}\left(Y_{\phi}\right)$ is torsion-free provided that $\operatorname{dim}\left(Y_{\phi}\right)=\operatorname{dim}(X) \geq 2$ (see for instance [1]). Then $q^{*}\left(P_{X}^{\prime}\right)=P_{Y_{\phi}}$ hence $\mathscr{C}_{\phi} \cong \mathscr{C}_{\phi^{\prime}}$ as in the previous proof.

4.3. Proof of the main result. We are now in position to prove our main theorem.

The map $F$ is defined on a dense open subset of $\mathscr{Y}$ as soon as there is $\phi \in \mathbf{W}$ such that $X_{\phi}$ has codimension $a-b+1$ unless $X_{\phi}$ is empty, and this is ensured by (2.1), which is clearly an open condition. By hypothesis, we have $\operatorname{dim}\left(X_{\phi}\right)=n+b-a-1 \geq 1$, so we can apply Lemma 4.2. We get that there are only finitely many G-orbits in the inverse image of a given point $\left[X_{\phi}\right] \in \mathscr{H}$, so that $F$ is generically finite, which proves (i). 
Next, assuming $\operatorname{dim}\left(X_{\phi}\right)=n+b-a-1 \geq 2$, by Proposition 3.1 and Lemma 2.1 we have $\operatorname{dim} \mathscr{T}_{\left[X_{\phi}\right], \mathscr{H}} \leq \operatorname{dim}(\mathscr{Y})$, and $\mathscr{Y}$ is irreducible and generically smooth. Since $F$ is generically finite, the image of $F$ also has dimension $\operatorname{dim}(\mathscr{Y}), \operatorname{so} \operatorname{dim} \mathscr{T}_{\left[X_{\phi}\right], \mathscr{H}}=$ $\operatorname{dim}(\mathscr{H})$ (i.e., the inequality in Proposition (3.1) is an equality). Hence $\mathscr{H}$ is generically smooth and $F$ is dominant on $\mathscr{H}$, so $\mathscr{H}$ is also irreducible. This proves (ii).

Finally, assuming $\alpha_{1}>\beta_{b}$ and $\operatorname{dim}\left(X_{\phi}\right) \geq 2, c=a-b+1 \geq 2$, we can apply Lemma 4.3. Then, by Lemma 4.1 the action of $\mathbf{G}$ is generically transitive on the set of determinantal representations of $X_{\phi}$, so $F$ is generically injective. Still $F$ is dominant by part (ii), hence $F$ is birational.

\section{Acknowledgments}

The authors would like to thank the referee for helpful comments.

D.F. partially supported by ANR contract Interlow ANR-09-JCJC-0097-0 and ANR GEOLMI.

M.L.F. partially supported by MIUR funds, PRIN 2010-2011 project "Geometria delle Varietà Algebriche."

\section{References}

[1] L. Bădescu, A remark on the Grothendieck-Lefschetz theorem about the Picard group, Nagoya Math. J. 71 (1978), 169-179.

[2] W. Bruns and U. Vetter, Determinantal rings, Lecture Notes Math., 1327, Springer-Verlag, Berlin, 1988, ISBN 3-540-19468-1

[3] M.C. Chang, A filtered Bertini-type theorem, J. Reine Angew. Math. 397 (1989), 214-219.

[4] L. Ein, On the cohomology of projectively Cohen-Macaulay determinantal subvarieties of $\mathbf{P}^{n}$, in 'Geometry of complex projective varieties (Cetraro, 1990)' (A. Lanteri, M. Palleschi, D. C. Struppa, eds.) 143-152, Sem. Conf., 9, Mediterranean, Rende, 1993.

[5] D. Eisenbud, Commutative algebra: with a view toward algebraic geometry, Graduate Texts in Mathematics, Vol. 150, Springer-Verlag, New York, 1995, ISBN 0-387-94268-8; 0-387-94269-6.

[6] G. Ellingsrud, Sur le schéma de Hilbert des variétés de codimension 2 dans $\mathbf{P}^{e}$ à cône de Cohen-Macaulay, Ann. Sci. École Norm. Sup. (4) 8(4) (1975), 423-431

[7] D. Faenzi and M.L. Fania, Skew-symmetric matrices and Palatini scrolls, Math. Ann. 347 (2010), 859-883, 10.1007/s00208-009-0450-5

[8] J. Harris, Algebraic geometry: a first course, Graduate Texts in Mathematics, Vol. 133, SpringerVerlag, New York, 1992, ISBN 0-387-97716-3.

[9] R. Hartshorne, Algebraic geometry, Graduate Texts in Mathematics, Vol. 52, Springer-Verlag, New York, 1977, ISBN 0-387-90244-9

[10] J.O. Kleppe, Deformations of modules of maximal grade and the Hilbert scheme at determinantal schemes, J. Algebra 407 (2014), 246-274.

[11] J.O. Kleppe, Families of low dimensional determinantal schemes, J. Pure Appl. Algebra 215(7) (2011), 1711-1725

[12] J.O. Kleppe, J.C. Migliore, R.M. Miró-Roig, U. Nagel and C. Peterson, Gorenstein liaison, complete intersection liaison invariants and unobstructedness, Mem. Amer. Math. Soc. 154(732) (2001), viii+116.

[13] J.O. Kleppe and R.M. Miró-Roig, Dimension of families of determinantal schemes, Trans. Amer. Math. Soc. 357(7) (2005), 2871-2907 (electronic)

[14] J.O. Kleppe and R.M. Miró-Roig, Families of determinantal schemes, Proc. Amer. Math. Soc. 139(11) (2011), 3831-3843

[15] R.M. Miró-Roig, Determinantal ideals, Progress in Mathematics, Vol. 264, Birkhäuser Verlag, Basel, 2008, ISBN 978-3-7643-8534-7

[16] D.G. Northcott, Finite free resolutions, Cambridge Tracts in Mathematics, Vol. 71, Cambridge University Press, Cambridge, 1976. 
[17] P. Pragacz, Enumerative geometry of degeneracy loci, Ann. Sci. École Norm. Sup. (4) 21(3) (1988), 413-454

[18] M. Rosenlicht, A remark on quotient spaces, An. Acad. Brasil. Ciênc. 35 (1963), 487-489.

[19] F. Tanturri, On the Hilbert scheme of degeneracy loci of twisted differential forms, 2014, arXiv e-print math.AG/1401.8188

[20] J. Vallès, Fibrés de Schwarzenberger et fibrés logarithmiques généralisés, Math. Z. 268(3-4) (2011), 1013-1023.

[21] J. Weyman, Cohomology of vector bundles and syzygies, Cambridge Tracts in Mathematics, Vol. 149, Cambridge University Press, Cambridge, 2003, ISBN 0-521-62197-6

Université de Pau et des Pays de l'Adour, Avenue de l'Université, BP 576, 64012 PAU Cedex, France

E-mail address: daniele.faenzi@univ-pau.fr

$U R L:$ http://univ-pau.fr/ faenzi/

Università degli Studi dell'Aquila, Via Vetoio Loc. Coppito, 67100 L'Aquila, Italy E-mail address: fania@univaq.it

URL: http://univaq.it/ fania/ 
